\title{
Networking Against Cancer
}

\author{
Norbert Marschner ${ }^{\mathrm{a}} \quad$ Martina Jänicke $^{\mathrm{b}}$ \\ ${ }^{a}$ Outpatient Centre for Interdisciplinary Oncology and Haematology, \\ biOMEDICO AG, Freiburg i.Br., Germany
}

The iOMEDICO Oncology Research Network connects physicians to facilitate access to clinical trials bringing research closer to patients. It was founded in 1994 by a group of oncologists to improve chances of participation in the competitive field of clinical research. The network currently links over 2,000 physicians working in almost 900 study sites at oncology outpatient centres, clinics, and universities spread across Germany (fig. 1).

The network conducts clinical trials of all phases in different tumour entities, including breast, colorectal, lung, renal, pancreatic, ovarian and haematological cancers. Further studies focus on supportive therapies, palliative care, quality of life, long-term side effects, and the role of physical activity and nutrition in cancer progression. The network is physicianled and physician-driven. Affiliated physicians take charge as coordinating and principal investigators, and many studies have been developed from ideas and initiatives within the network. Periodic advisory boards and regular tumour entity specific meetings discuss new ideas, general strategies and ongoing studies. Every study site interested in recruiting patients for one of the network's studies can join the network and benefit from its infrastructure. Over the years, successful working relationships have been formed with numerous study groups, non-commercial and pharmaceutical partners. In March 2011, almost 20,000 patients were participating in the network's interventional and non-interventional studies.

\section{Research for Rare Patient Populations at Oncology Outpatient Centres}

'New structures for improved health care' is the motto of the new concept 'Kolibri' that was launched by the network at the end of 2010 in cooperation with the Arbeitskreis Klinische Studien (working group for clinical studies, AKS). Kolibri aims to facilitate clinical trials for rare patient populations at local oncology outpatient centres. Single outpatient centres are usually excluded from participation in such trials, because they treat only few patients fulfilling inclusion criteria such as a specific molecular tumour profile or a rare cancer entity. As a consequence these patients do not have access to clinical trials or have to travel long distances to receive optimal care, adding additional burden to patients and care giving relatives.

The key idea of Kolibri is that 2-5 local oncology outpatient centres collaborate in 'micro circles'. Within each micro circle the centres take turns at being study site of a 'Kolibri trial', and patients potentially eligible for a Kolibri trial are transferred to the respective study site (fig. 2). All trial specific procedures are performed at the study site. Once the trial is completed, patients continue their treatment at the original outpatient centre.

Equal allocation of trials between all micro circles, alternating selection of study sites within micro circles and an independent coordinating central service provider, the iOMEDICO AG, ensure fairness and transparency within Kolibri. Regular meetings discuss ongoing trials, and a scientific advisory board assists with the planning of trials suitable for Kolibri. The first Kolibri trials started in 2011 with 50 outpatient centres forming 17 micro circles spread across Germany.

\section{Infrastructure of the Oncology Research Network}

From the beginning the network has worked with the iOMEDICO AG as the central service provider. iOMEDICO serves as a mediator between the network and interested sponsors, and has itself been sponsor for multiple network-initiated trials. Its team provides technology, research management, and quality assurance as well as logistic, administrative and personnel support.

A complex intranet-based technology platform was developed to link the network partners offering free electronic tools for data collection, information, and benchmarking (fig. 3). Patient data are entered into electronic case report

\section{KARGER}

Fax +497614520714

Information@Karger.de

www.karger.com (c) 2011 S. Karger GmbH, Freiburg

1661-3791/11/0063-0245\$38.00/0

Accessible online at:

www.karger.com/brc
Dr. med. Norbert Marschner

Praxis für interdisziplinäre Onkologie und Hämatologie

Breisacherstr. 117

79106 Freiburg i.Br., Germany

norbert.marschner@onkologie-freiburg.de 


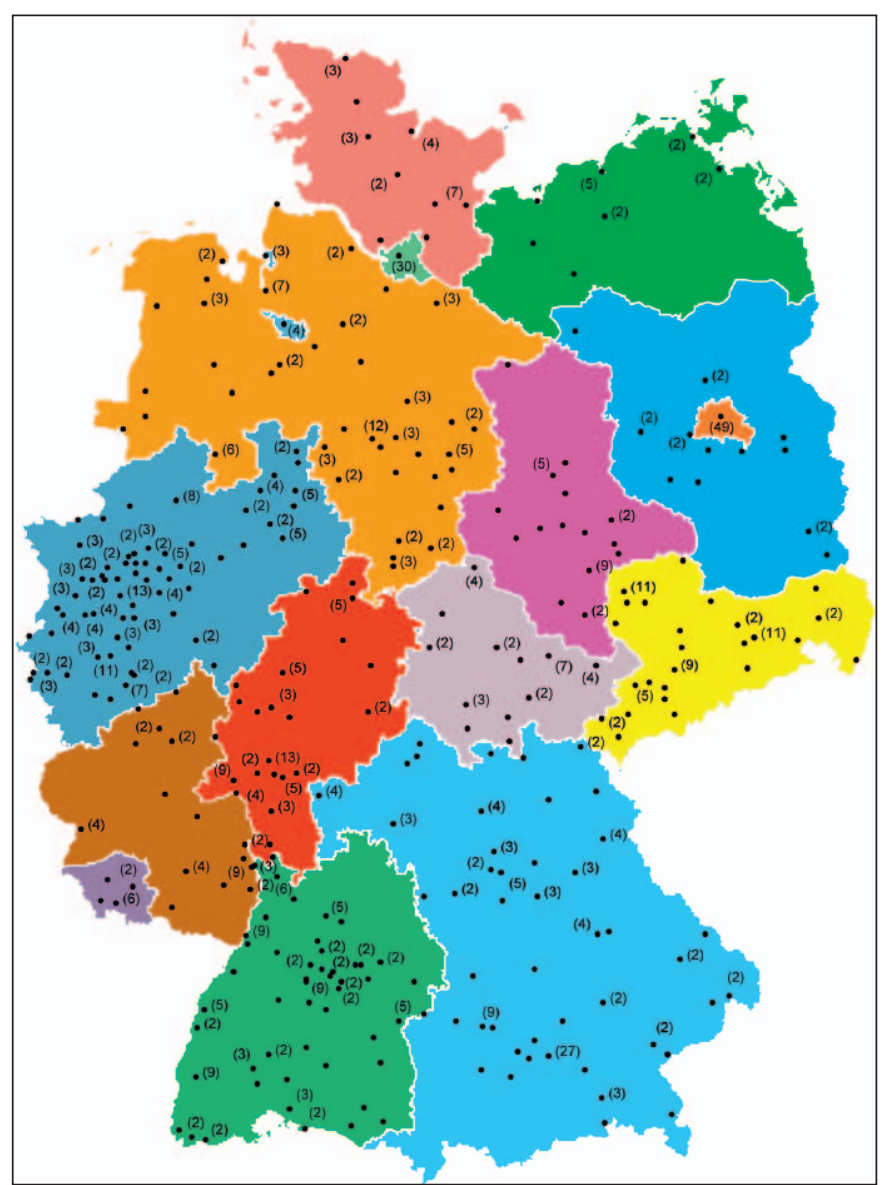

Fig. 1. Location of study sites participating in the network.

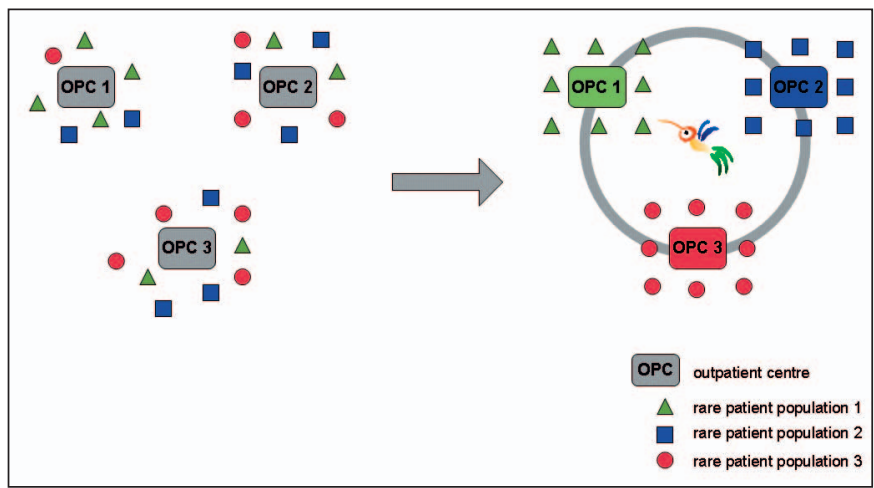

Fig. 2. The Kolibri concept - local outpatient centres collaborate in micro circles.

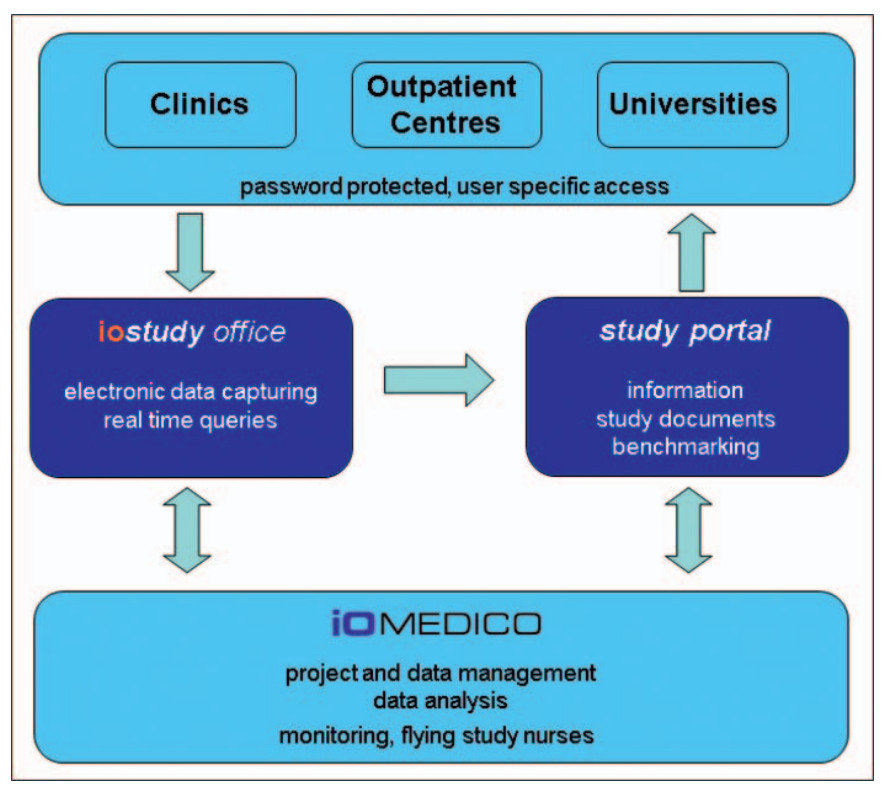

Fig. 3. The network's infrastructure.

\section{Breast Cancer Research within the Network}

forms (eCRFs) of the network's electronic documentation software. Implemented completeness and plausibility checks generate queries in real time and allow immediate resolving. User and study site specific information about projects and study documents are accessed through the study portal. A benchmarking system displays key variables of a project in a user-specific, anonymous manner in comparison to all participating sites.

iOMEDICO coordinates all of the network's studies. Study sites receive maximal support in the conduction of trials and the collection of data. Standardised processes facilitate data collection and reduce the time required to initiate new studies because study sites are familiar with procedures and quality standards. If requested, 'flying study nurses' can assist with documentation and organisation in study sites. Project and data management, monitoring, coordination of finances as well as data analysis are central services provided.
Almost 400 study sites from the network participate in interventional and non-interventional studies with breast cancer patients. In March 2011, 5,300 patients with breast cancer were participating in the network's studies. Two examples shall highlight this work: the CARIN trial and the Clinical Breast Cancer Registry.

The CARIN trial was initiated by investigators from the network to identify a safe and effective non-taxane, alopeciasparing regimen for women with metastatic or advanced, HER2-negative breast cancer. Such a regimen would provide an alternative with acceptable tolerability to the renewed application of taxanes and anthracylines. The randomised, controlled phase IIb trial tests superiority of the triple combination capecitabine, bevacizumab and vinorelbine compared to capecitabine and bevacizumab alone. The first patient was enrolled in February 2009 and within 23 months all 400 patients were recruited by the 60 participating study sites. First 
safety results will be presented at the American Society of Clinical Oncology Meeting in 2011.

The Clinical Breast Cancer Registry was started by the network in 2007 in cooperation with the AKS. The aim of the registry is to analyse the 'real life' health care setting for patients with breast cancer in Germany. Patients are recruited at the start of their (neo)adjuvant or first-line palliative treatment, independent of age or comorbidities and are followed for a maximum of 5 years. For every patient the registry collects data on all treatment regimens; doses, duration and effectiveness of treatment; demographic and tumour characteristics; biological predictors; comorbidities; side effects of key substances; radiotherapies and surgeries; follow up exams; pattern of metastases; decision basis for the treatment chosen; and participation in clinical trials. Within 3 years, over 2,500 patients have been recruited by the 100 participating study sites and another 2,000 patients will be recruited by 2014 .

The registry data will allow to assess the effectiveness (efficacy in routine practice) of treatments as well as to investigate sequences of regimens across lines of therapy. The effect of comorbidities on the course of the disease will be analysed and prognostic scores developed.

Fast and successful execution of numerous clinical research projects has demonstrated that a physician-driven oncology research network is beneficial for all parties involved - patients get access to investigational treatments, physicians broaden their expertise in novel therapies, sponsoring partners save time and costs, and researchers gain new insights into cancer therapy. 